\title{
Long non-coding RNA PCAT-1 promotes tumor progression by inhibiting miR-129-5p in human ovarian cancer
}

Li-Ping Gu, Shuo Jin, Rong-Chun Xu, Jing Zhang, Ying-Chun Geng, Xing-Yue Shao, Li-Bo Qin

Department of Obstetrics-Gynecology, Daqing Oil Field General Hospital, Daqing, Heilongjing, China

Submitted: 24 November 2017

Accepted: 19 December 2017

Arch Med Sci 2019; 15, 2: 513-521

DOI: https://doi.org/10.5114/aoms.2018.75534

Copyright $\odot 2018$ Termedia \& Banach

\section{Abstract}

Introduction: Ovarian cancer $(\mathrm{OC})$ is one of the most common malignancies and the leading cause of cancer-related death among women. The long non-coding RNA Prostate cancer-associated transcript-1 (PCAT-1) has been reported to play important roles in multiple human cancers. However, the role of PCAT- 1 in OC has never been investigated. The purpose of this study was to investigate the expression and roles of PCAT-1 in OC.

Material and methods: Expression of PCAT-1 and miR-129-5p in OC tissues and cell lines was determined by qRT-PCR. Cell proliferation and apoptosis were analyzed by MTT assay and flow cytometry, respectively. The interaction between PCAT-1 and miR-129-5p was demonstrated by luciferase reporter assay. Results: PCAT-1 is significantly upregulated in OC tissues and cell lines ( $p<$ 0.05 ). Overexpression of PCAT- 1 promotes proliferation of OC cells and inhibits their apoptosis $(p<0.05)$. In addition, miR-129-5p is markedly downregulated in OC and its level is inversely correlated with PCAT- 1 expression in OC tumor tissues $(p<0.05)$. miR-129-5p inhibits proliferation and induces apoptosis in OC cell lines $(p<0.05)$. Furthermore, it has been demonstrated that miR-129$5 p$ is directly targeted by PCAT- 1 and miR-129-5p overexpression can effectively attenuate the effects of PCAT- 1 on the proliferation and apoptosis of OC cells. Conclusions: Our results suggest that PCAT-1 functions as an oncogene by inhibiting miR-129-5p in OC and silencing PCAT-1 may be a novel therapeutic strategy in the treatment of OC.

Key words: ovarian cancer, long non-coding RNA, prostate cancer-associated transcript-1, miR-129-5p.

\section{Introduction}

Ovarian cancer $(\mathrm{OC})$ is one of the most common malignancies and the leading cause of cancer-related death among women [1, 2]. Each year, more than 230,000 new cases of OC are diagnosed and this disease causes at least 140,000 deaths in women worldwide [3]. Despite the advances in the detection and treatment of ovarian cancer during the last two decades, the prognosis is still very poor and it remains the fifth leading cause of cancer-associated mortality in women $[4,5]$. Thus, it is urgent to identify novel molecules involved in the progression of ovarian cancer and to develop some other effective treatments for OC patients.

Long non-coding RNAs (IncRNAs) are a class of RNAs with more than 200 nucleotides in length and have little protein coding potential $[6,7]$.

\author{
Corresponding author: \\ Dr. Rong-Chun Xu \\ Department of \\ Obstetrics-Gynecology \\ Daqing Oil Field \\ General Hospital \\ 9 Zhongkang St \\ Daqing, Heilongjing \\ 163316, China \\ Phone: +8604595805999 \\ Fax: +8604595805999 \\ E-mail: chunrongxu1@163. \\ com
}


Prostate cancer-associated transcript-1 (PCAT-1) is a novel IncRNA which was originally discovered in prostate cancer [8]. The biological functions of PCAT-1 have been documented in multiple human cancers. For example, Bi et al. found that PCAT-1 is increased in gastric cancer and the knockdown of PCAT-1 inhibited the proliferation of gastric cancer cells [9]. PCAT-1 was also demonstrated to act as an oncogene in hepatocellular carcinoma and colorectal cancer [10, 11]. However, the expression and functions of PCAT- 1 in ovarian cancer have never been investigated.

In this study, we firstly found that PCAT-1 contained a similar theoretical combing site for miR-129-5p by bioinformatics analysis. The expression of PCAT-1 and miR-129-5p in OC was detected by qRT-PCR. Then, the biological functions of PCAT-1 and miR-129-5p on OC cells were determined. Moreover, we verified that PCAT-1 can directly target miR-129-5p and negatively regulate its expression in OC. Overexpression of miR129-5p partly reversed the effects of PCAT- 1 on OC cells. Collectively, we demonstrated that long non-coding RNA PCAT-1 promotes tumor progression by inhibiting miR-129-5p in human ovarian cancer and PCAT-1 may function as a therapeutic target in the treatment of OC.

\section{Material and methods}

Tissue samples and cell culture

A total of 30 ovarian cancer tissues and matched adjacent normal tissues were obtained from Obstetrics-Gynecology, Daqing Oil Field General Hospital. Tissue samples were frozen immediately in liquid nitrogen and stored until used. Every patient signed the informed consent prior to enrolling in the study. This study was supported by the Ethics Committee of Daqing Oil Field General Hospital. This study was performed following the Declaration of Helsinki. Human OC cell lines (A2780, SK-OV-3, OVCA429, and HO8910) and the human normal ovarian cell line IOSE80 were purchased from the American Type Culture Collection (ATCC, VA, USA). Cells were cultured in DMEM supplemented with $10 \%$ fetal bovine serum (FBS) (both from Gibco, CA, USA). Cells were all maintained in a humidified incubator containing $5 \% \mathrm{CO}_{2}$ at $37^{\circ} \mathrm{C}$.

\section{RNA extraction and qRT-PCR}

Total RNA was extracted from tissue samples or cell lines using TRIzol reagent (Invitrogen, Carlsbad, CA, USA) following the manufacturer's instructions. CDNA was reverse transcribed using M-MLV (Thermo Fisher Scientific, Inc., Rockford, IL, USA) containing $5 \mu \mathrm{g}$ of total RNA. qRT-PCR was performed using SYBR Green PCR
Master mix (Thermo Fisher Scientific, Inc.) on an ABI 7300 machine (Applied Biosystems, Foster City, CA, USA). The relative expression was calculated and normalized using the $2^{-\Delta \Delta c t}$ method. GAPDH and U6 were used as an internal control for PCAT-1 and miR-129-5p, respectively. The primer sequences were as follows: PCAT-1 forward: 5'-AATGGCATGAACCTGGGAGGCG-3' and reverse: 5'-GGCTTTGGGAAGTGCTTTGGAG-3'; miR-129-5p forward: 5'-ACACTCCTTTTTGCGTCTGGGCTTGC-3' and reverse: 5'-TGGTGTCGTGGAGTCG-3'; GAPDH forward: 5'-CACCCACTCCTCCACCTTTG-3' and reverse: 5'-CCACCACCCTGTTGCTGTAG-3'; U6 forward: 5'-TTACATTGCTATCCACAGAACGG-3' and reverse $5^{\prime}$ - CTATGCTGCTGCTTTTTGCTC-3'. This experiment was repeated three times.

\section{Cell transfection}

Cell transfections were all performed using Lipofectamine 2000 (Invitrogen) according to the manufacturer's protocol. To overexpress PCAT-1 in OC cells, the CDNA of PCAT- 1 was cloned into the pcDNA3.1 vector (Invitrogen). The empty vector was used as a control. To downregulate PCAT-1, small interfering RNA targeting PCAT-1 (si-PCAT-1) or the control si-NC was transfected into OC cells. The sequences of si-PCAT-1 were: $5^{\prime}$-AUACAUAAGACCAUGGAAATT-3' (sense) and 5'-UUUCCAUGGUCUUAUGUAUTT-3' (antisense); siRNAs, microRNA-129-5p mimics, inhibitors and the negative controls were all synthesized by GenePharma Co. Ltd (Shanghai, China). The transfect efficiency was assessed following $48 \mathrm{~h}$ of transfection.

\section{MTT assay}

After effective transfection, cells were seeded into 96 -well plates and cultured at $37^{\circ} \mathrm{C}$. Cell viability was measured at various time points $(0 \mathrm{~h}$, $24 \mathrm{~h}, 48 \mathrm{~h}$ and $72 \mathrm{~h}$ after seeding). Cell viability was analyzed by the 3-(4,5-dimethylthiazol-2-yl)-2, 5-diphenyl tetrazolium bromide (MTT) assay according to the manufacturer's instructions. In brief, at each time point, $20 \mu \mathrm{l}$ MTT $(5 \mathrm{mg} / \mathrm{ml}$, Sigma) was added into each well and cultured for a further $4 \mathrm{~h}$. Then, the media were discarded and $150 \mu$ l of DMSO (Sigma) was added to each well. The absorbance at $490 \mathrm{~nm}$ was measured by a microplate reader (Bio-Rad).

\section{Apoptosis assay}

Cell apoptosis rate was determined by flow cytometry with the FITC-Annexin V Apoptosis Detection Kit (BD Biosciences, San Jose, CA) according to the manufacturer's protocol. Cells were collected, stained with Annexin $\mathrm{V}$ and PI for 15 min in the dark, and then analyzed by a FACS Caliber flow cytometer (BD Bioscience). 


\section{Luciferase reporter assay}

For the luciferase reporter assay, the oligonucleotides of the PCAT-1 fragment containing wildtype (WT) and mutant (mut) miR-129-5p binding sites were amplified and cloned into the PGL3 vector (Promega, Madison, WI, USA) to construct reporter plasmids. The day before transfection, 293T cells were seeded in 24-well plates and cultured at $37^{\circ} \mathrm{C}$. Subsequently, cells were transfected with wild-type (WT) or mutated (mut) PCAT-1 reporter plasmids and miR-129-5p mimics (miR-129) or negative control (miR-NC) using Lipofectamine 2000 (Invitrogen). After $48 \mathrm{~h}$, the firefly luciferase activity was examined and normalized to that of Renilla using the Dual-Luciferase Reporter Assay System (Promega, Madison, WI, USA).

A

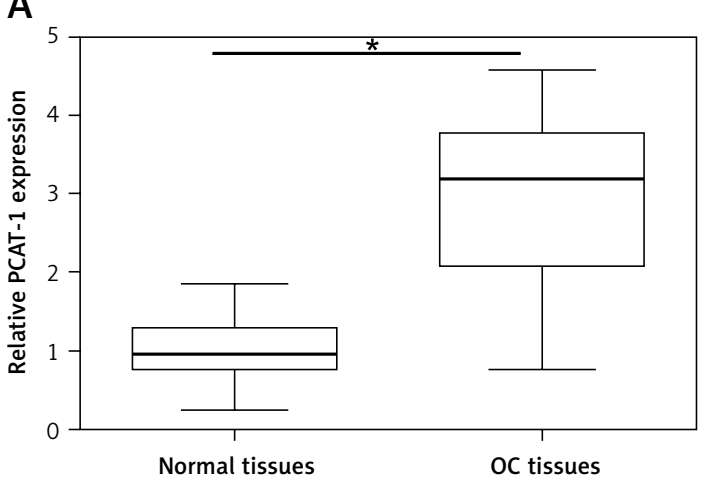

C

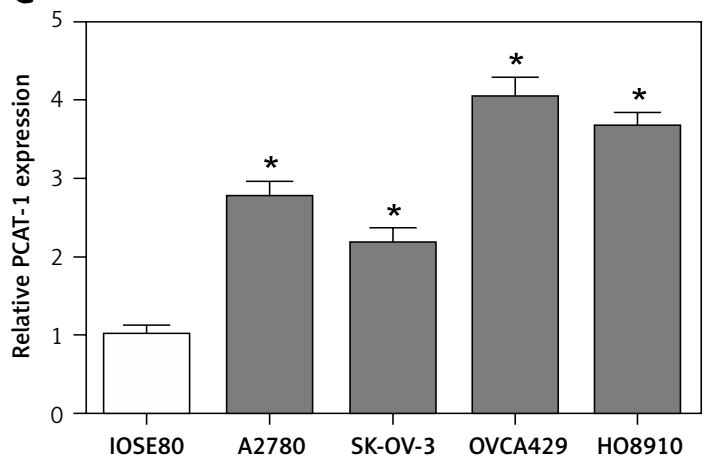

$\mathrm{E}$

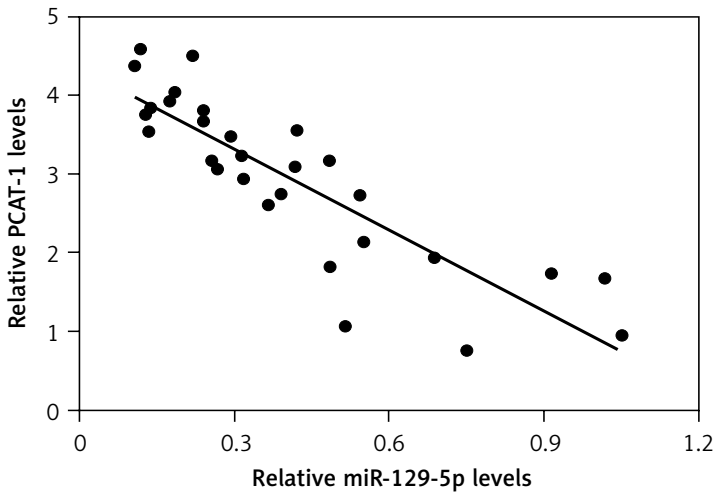

\section{Statistical analysis}

Data were expressed as mean \pm standard deviation (SD). Statistical analysis was performed using GraphPad Prism 6.0 software (San Diego, CA). Differences between groups were analyzed using Student's $t$ test or one-way analysis of variance. Results were considered significant when the $p$-value was less than 0.05 .

\section{Results}

\section{PCAT-1 is upregulated and negatively} correlated with miR-129-5p in OC

First, we detected the expression levels of long non-coding RNA PCAT-1 and miR-129-5p in human ovarian cancer tissues and matched nor-
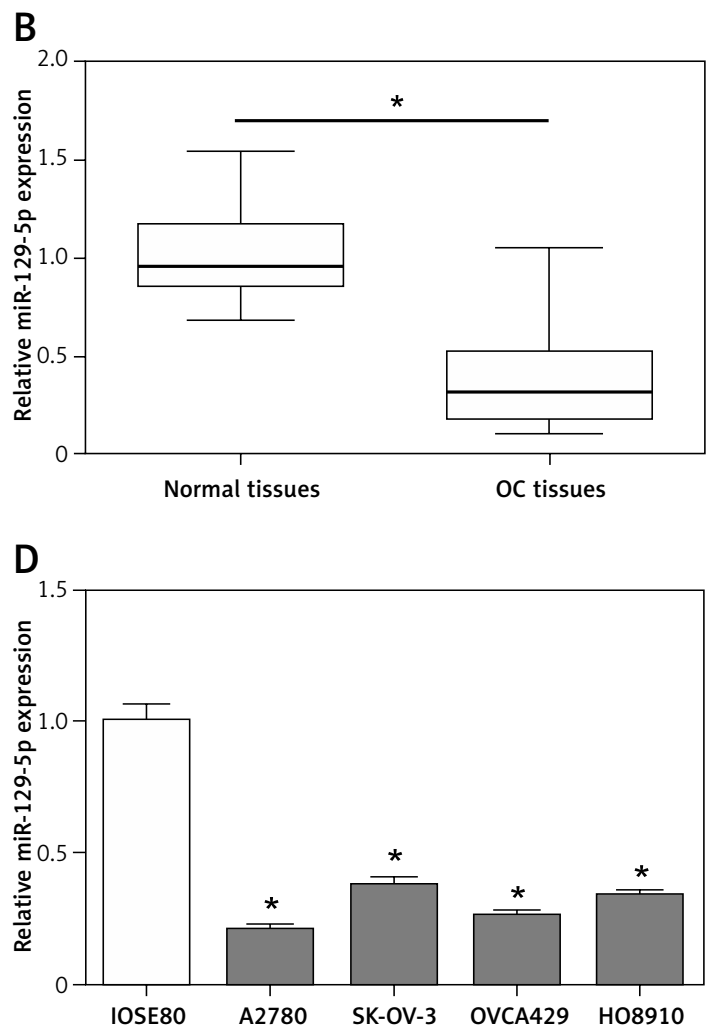

Figure 1. PCAT-1 is upregulated and negatively correlated with miR-129-5p in OC. A, B - Analysis of long non-coding RNA PCAT-1 (A) and miR-129$5 p$ (B) expression levels in OC tumor tissues and the corresponding normal tissues by qRT-PCR. C, D - Analysis of PCAT-1 (C) and miR-129-5p (D) expression levels in OC cell lines (A2780, SK-OV-3, OVCA429, and H08910) and the human normal ovarian cell line IOSE80 by qRT-PCR. E - The correlation analysis between PCAT-1 and miR-129$5 p$ levels in OC tumor tissues was performed by GraphPad Prism 6

Data are mean $\pm S D .{ }^{*} P<0.05$. 

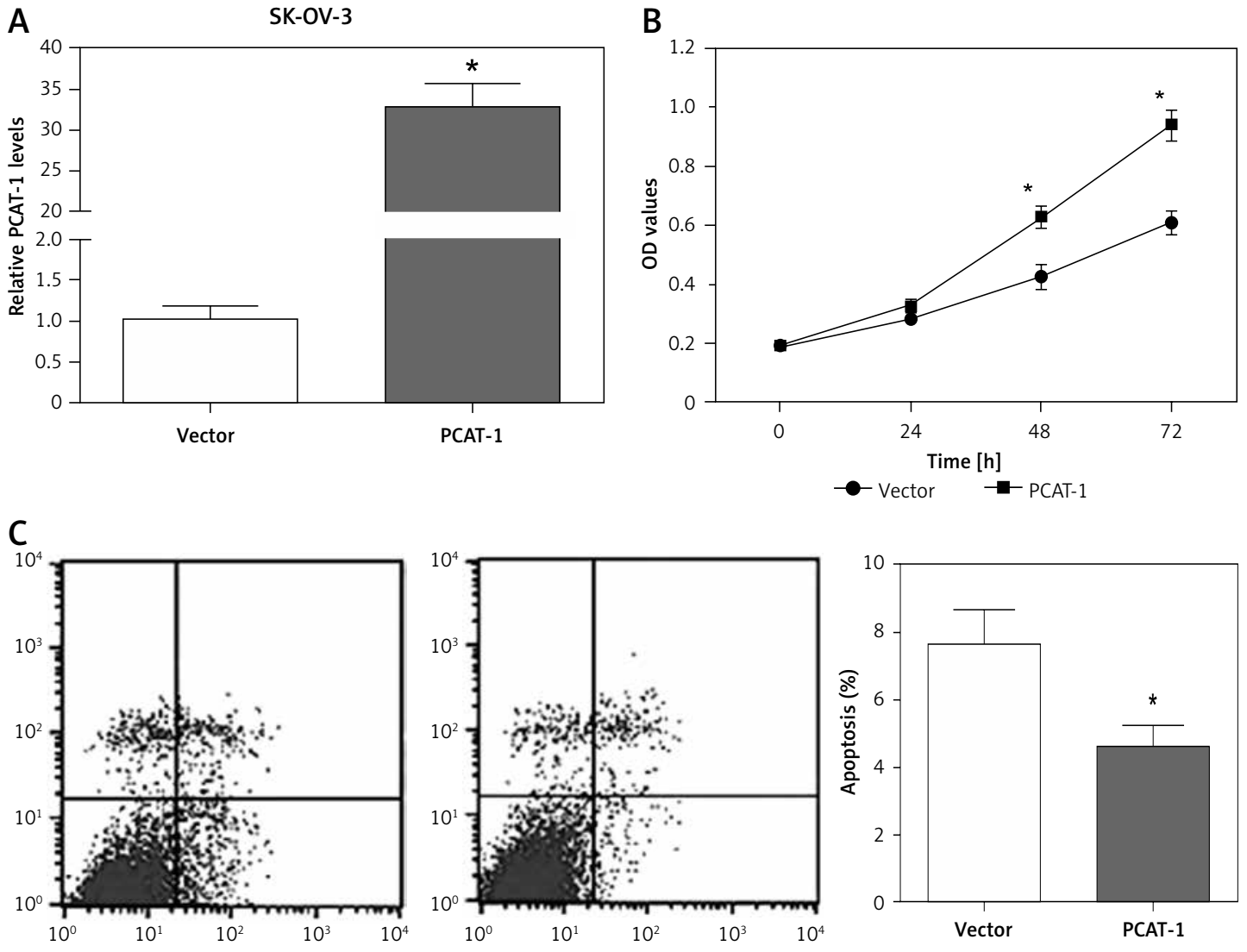

D

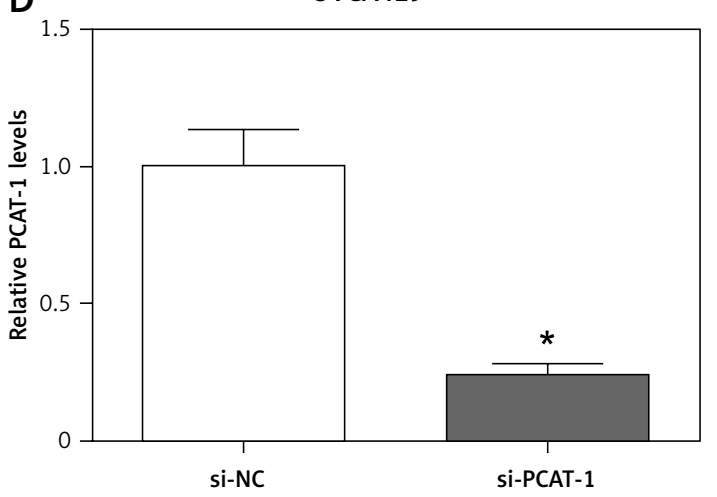

$\mathrm{E}$
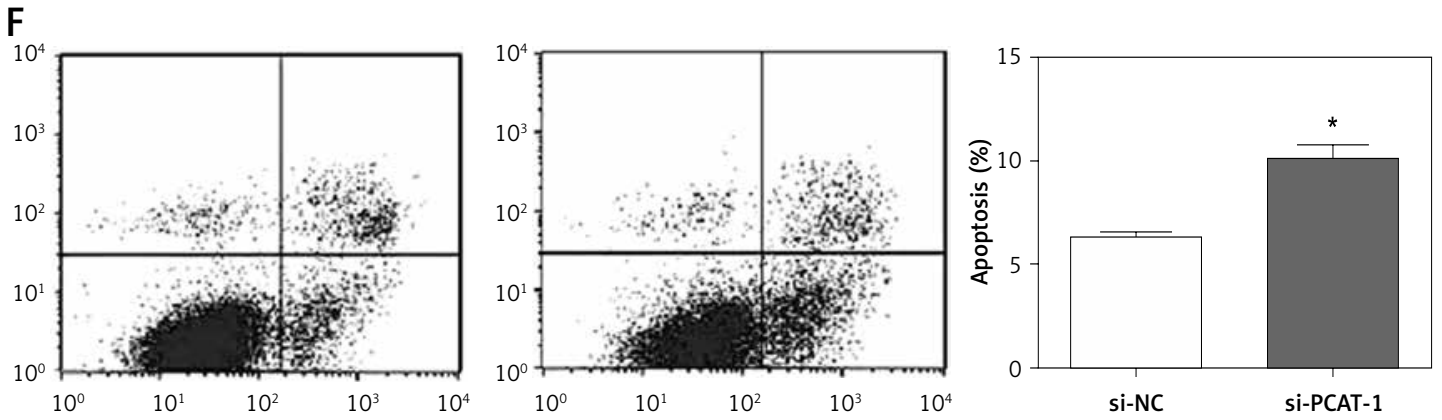

Figure 2. PACT-1 promotes proliferation but inhibits apoptosis in OC cells. A - The efficiency of PCAT-1 overexpression by pcDNA3.1-PCAT-1 plasmid (PCAT-1) in OC cells SK-OV-3. The empty vector (vector) was used as a control. B - Proliferation of SK-OV-3 cells after PCAT-1 overexpression. C - Apoptosis rate of SK-OV-3 cells after PCAT-1 overexpression. D - Efficiency of PCAT-1 knockdown by siRNA (si-PCAT-1) in OVCA429 OC cells. The negative control is si-NC. E - Proliferation of OVCA429 cells after PCAT-1 knockdown. F - Apoptosis rate of OVCA429 cells after PCAT-1 knockdown Data are mean $\pm S D .{ }^{*} P<0.05$. 
mal tissues using qRT-PCR. The data showed that PCAT-1 is significantly upregulated while miR-129$5 p$ is downregulated in OC tissues compared with adjacent normal tissues (Figures $1 \mathrm{~A}, \mathrm{~B}$ ). Then, the expression patterns of PCAT-1 and miR-129$5 p$ were also examined in OC cell lines (A2780, SK-OV-3, OVCA429, and H08910) and the human normal ovarian cell line IOSE80. The results of qRT-PCR indicated that the expression of PCAT-1 is increased while miR-129-5p is decreased in OC cell lines in comparison with the human normal ovarian cell line IOSE80 (Figures 1 C, D). Moreover, we observed that the level of PCAT- 1 is inversely correlated with miR-129-5p expression in ovarian cancer tissues (Figure $1 \mathrm{E}$ ). These results suggest that PCAT-1 and miR-129-5p are involved in the development of ovarian cancer.

\section{PACT-1 promotes proliferation but inhibits apoptosis in OC cells}

Considering the high expression of PCAT-1 in OC, we determined the biological effects of PCAT-1 on OC cells. The SK-OV-3 OC cells were transfected with pcDNA3.1-PCAT-1 plasmid to overexpress PCAT-1 (Figure 2 A). Then, MTT assay and flow cytometry were conducted to analyze cell proliferation and apoptosis. The results showed that overexpression of PCAT-1 significantly promoted proliferation but inhibited apoptosis in SK-OV-3 cells (Figures 2 B, C). Conversely, si-PCAT- 1 was transfected into OVCA429 cells to downregulate the level of PCAT-1 (Figure 2 D). MTT assay and apoptosis analysis indicated that knockdown of PCAT-1 suppressed proliferation while inducing apoptosis in OVCA429 cells (Figures 2 E, F). These data demonstrate that PCAT- 1 functions as an oncogene in the development of OC.

\section{miR-129-5p inhibits proliferation and induces apoptosis in OC cells}

The expression of miR-129-5p is downregulated in OC tissues and cell lines, indicating its potential role in OC development. miR-129-5p mimics were transfected into OVCA429 OC cells to overexpress miR-129-5p (Figure $3 \mathrm{~A}$ ). We then determined the effects of miR-129-5p mimics on OVCA429 cell proliferation and apoptosis. The data showed that miR-129-5p mimics significantly inhibited proliferation and induced apoptosis in OVCA429 cells (Figures 3 B, C). In contrast, miR-129-5p inhibitors were used to suppress its expression in the OC cell line SK-OV-3 (Figure $3 \mathrm{D})$. We found that miR-129-5p suppression enhanced the proliferation ability and decreased the apoptosis rate in SK-OV-3 cells (Figures 3 E, F). These results reveal that miR-129-5p acts as a tumor suppressor in OC.

\section{PACT-1 exerts its functions by directly targeting miR-129-5p in OC}

As shown above, the expression of miR-129-5p is inversely correlated with PCAT-1 in OC tissues. PCAT-1 functions as an oncogene while miR-129$5 p$ acts as a tumor suppressor in OC development. We wondered whether PCAT-1 directly targets and regulates miR-129-5p in OC. The bioinformatics database Starbase v2.0 was used to search for candidate miRNAs that can bind to PCAT-1. We found that miR-129-5p can directly bind to PCAT-1 (Figure $4 \mathrm{~A}$ ). To confirm the interaction between miR-129-5p and PCAT-1, the luciferase reporter assay was performed. We observed that miR-129$5 p$ mimics significantly decreased the luciferase activity of the wild type (WT) but not mutant (mut) PCAT-1 reporter system (Figure 4 B). The expression of miR-129-5p in OC cells was detected following PCAT-1 overexpression or knockdown. The data showed that miR-129-5p is decreased after PCAT-1 overexpression and increased after PCAT-1 knockdown in OC cells (Figure 4 C). Furthermore, miR-129-5p restoration attenuated the effects of PCAT-1 overexpression on SK-OV-3 cell proliferation and apoptosis (Figures $4 \mathrm{D}, \mathrm{F}$ ). Conversely, miR-129-5p suppression partly abolished the effects of PCAT-1 knockdown on OVCA429 cell proliferation and apoptosis (Figures 4 E, F). These results suggest that PACT-1 can directly target miR-129-5p and negatively regulate its expression to promote tumor progression in OC.

\section{Discussion}

Recent studies have reported that IncRNAs have multiple biological functions in various stages of OC development. It has been concluded that IncRNAs are closely involved in the pathogenesis of OC; and the expression of IncRNAs indicates the early diagnosis, prognosis, and therapeutic response of OC cells [12]. Numerous IncRNAs were reported to be deregulated in ovarian cancer. For instance, Li et al. showed that IncRNA GAS5 was markedly decreased in OC tissues and patients with low GAS5 expression had poorer prognosis [13]. LncRNA SPRY4-IT1 was reported to be upregulated in ovarian tumor tissues and OC cell lines. The knockdown of IncRNA SPRY4-IT1 arrested the cell cycle at the G0/G1 stage in OC cells and thus inhibited the proliferation of OC cells [14]. Wu et al. demonstrated that InCRNA MALAT1 was upregulated in OC. Knockdown of MALAT1 repressed growth and migration of OC cells [15]. LncRNA PCAT-1 was reported to play roles in multiple human cancers, but the effects of PCAT-1 in ovarian cancer have never been reported.

In the current study, qRT-PCR assays showed that PACT-1 expression was significantly upregu- 
A

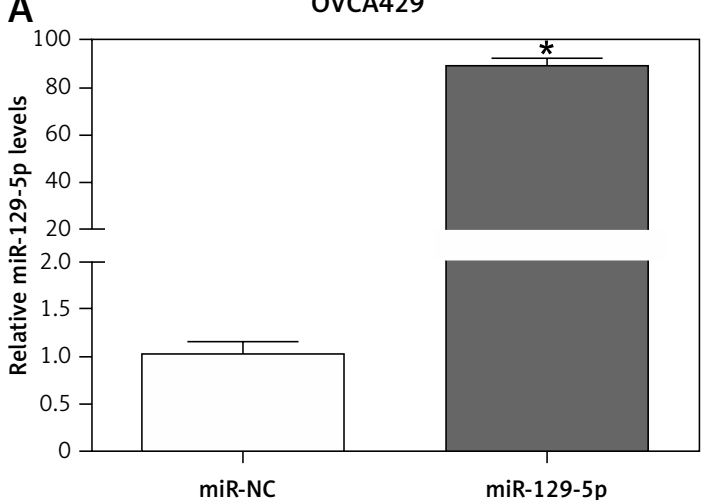

B

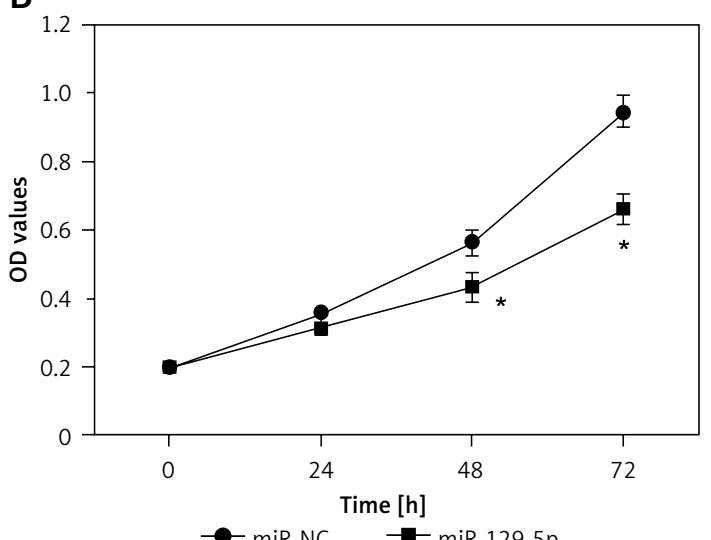

C

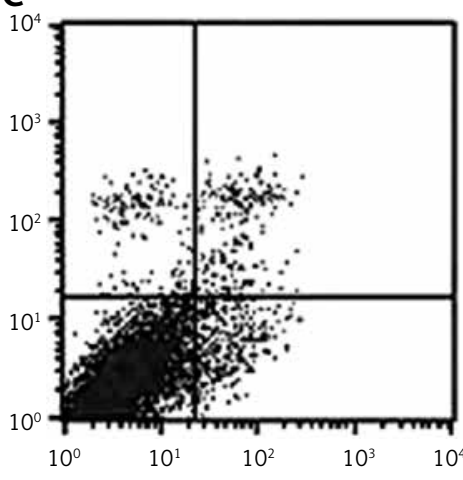

D

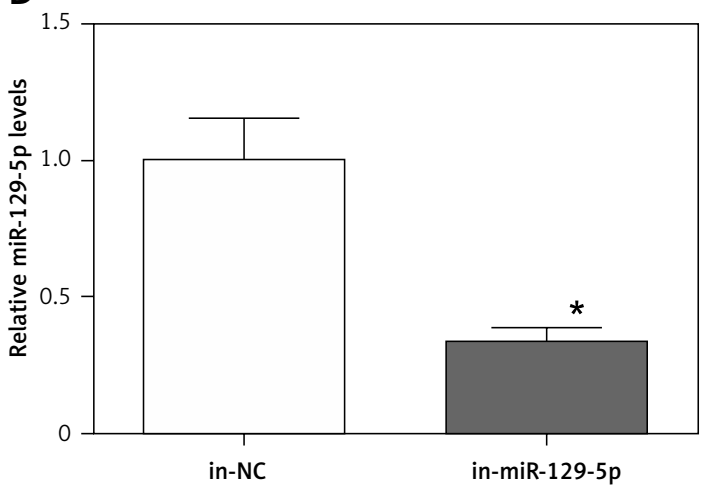

$\mathrm{E}$
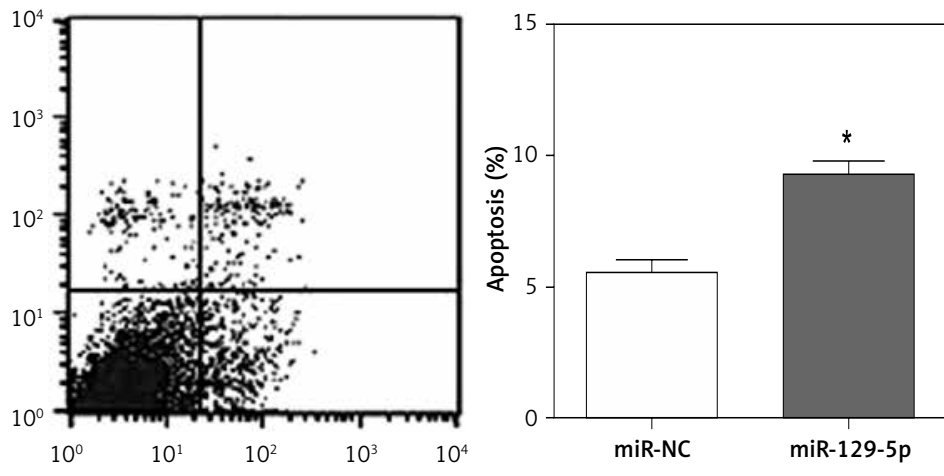

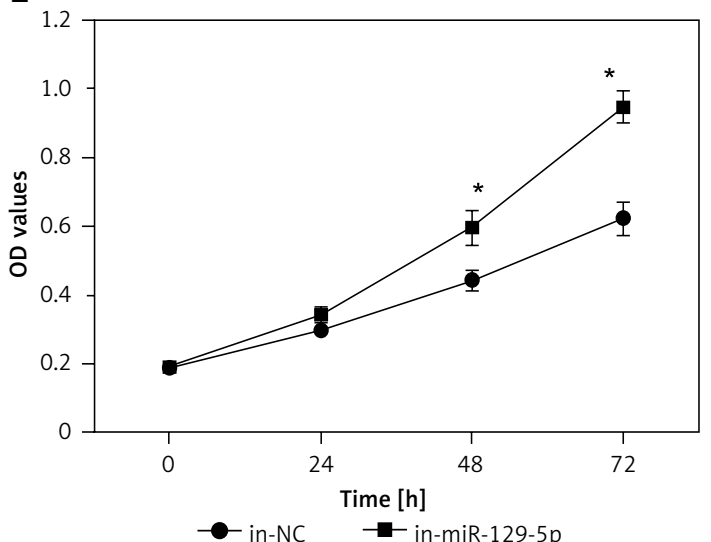

$\mathrm{F}$
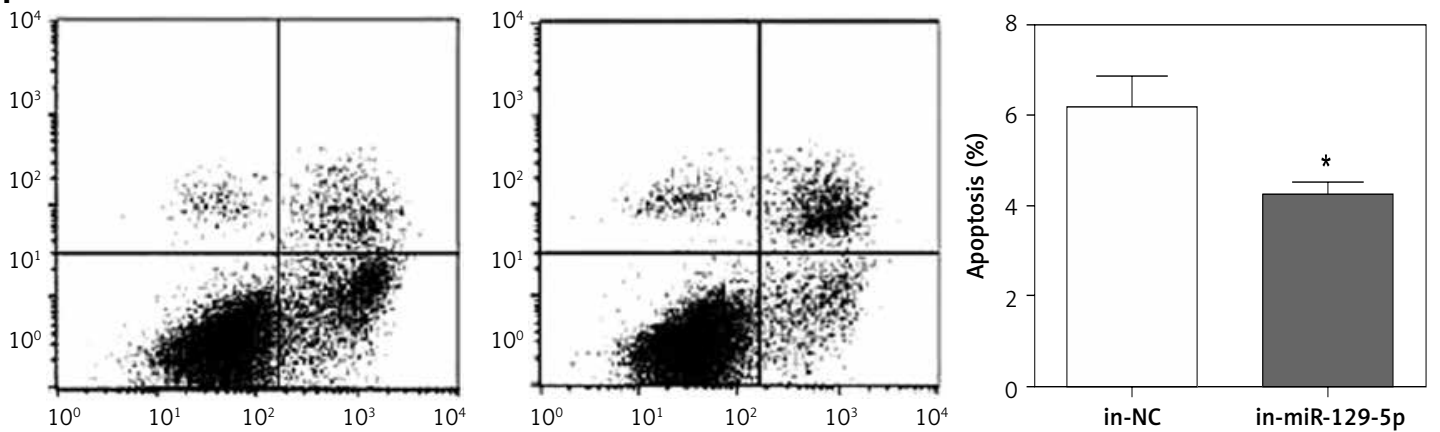

Figure 3. miR-129-5p inhibits proliferation and induces apoptosis in OC cells. A - Overexpression of miR-129-5p by its microRNA mimics (miR-129-5p) in OC cells OVCA429. miR-NC was used as a negative control. B - Proliferation of OVCA429 cells after miR-129-5p overexpression. C - Apoptosis rate of OVCA429 cells after miR-129-5p overexpression. D - miR-129-5p suppression by its microRNA inhibitors (in-miR-129-5p) in OC cells SK-OV-3. The negative control is in-NC. E - Proliferation of SK-OV-3 cells after miR-129-5p suppression. F - Apoptosis rate of SK-OV-3 cells after miR-129-5p suppression

Data are mean $\pm S D .{ }^{*} P<0.05$. 
lated while miR-129-5p was significantly downregulated in OC tumor tissues and the expression of miR-129-5p was inversely correlated with PCAT-1. To determine the biological functions of PCAT-1 in OC development, the level of PCAT-1 in OC cells was overexpressed or suppressed by transfection with pcDNA3.1-PCAT-1 plasmid or siRNA.

A

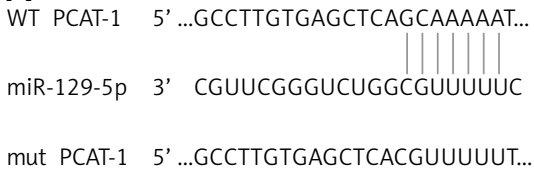

$\mathrm{C}$

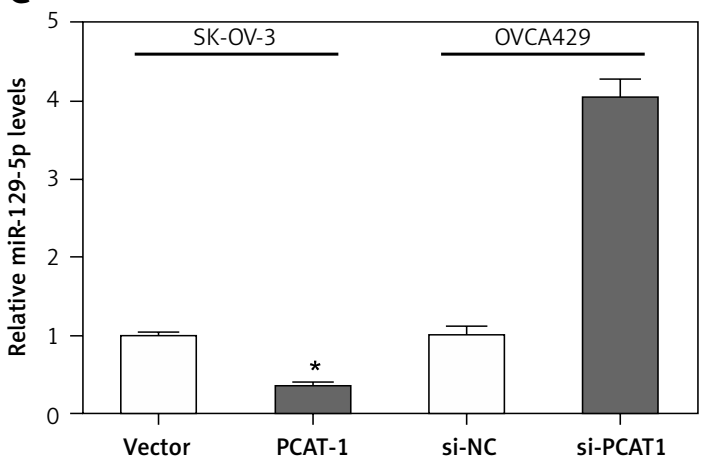

E

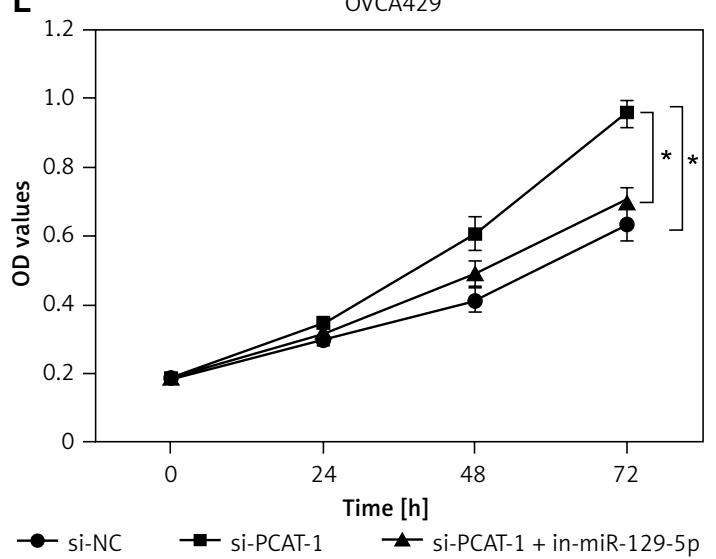

Functional assays demonstrated that PACT-1 promoted proliferation but inhibited apoptosis in OC cells. Furthermore, the effects of miR-129-5p in OC were also studied. We found that overexpression of miR-129-5p significantly inhibited cell proliferation and induced apoptosis in OC cells; suppression of miR-129-5p exhibited opposite

B

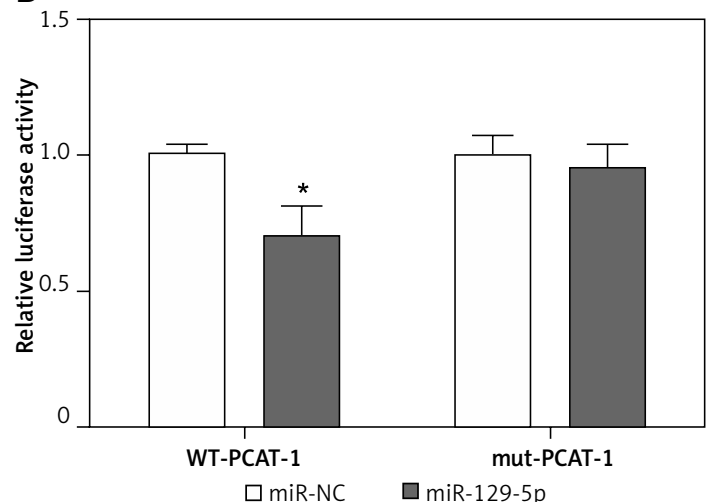

D

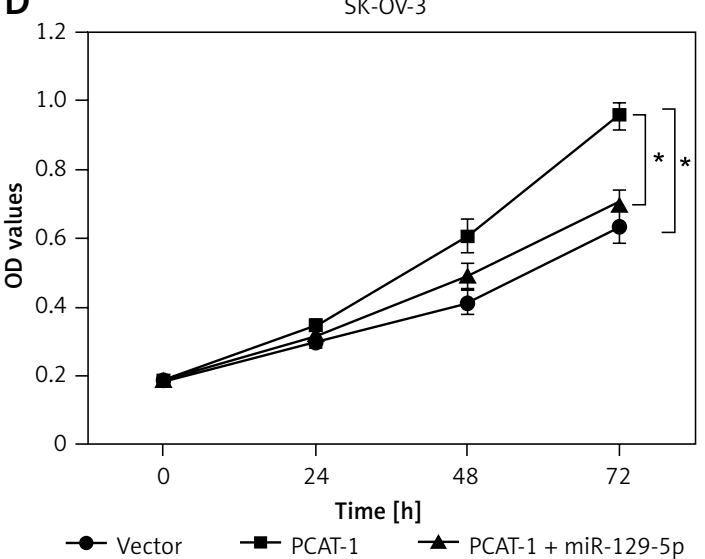

$\mathbf{F}$

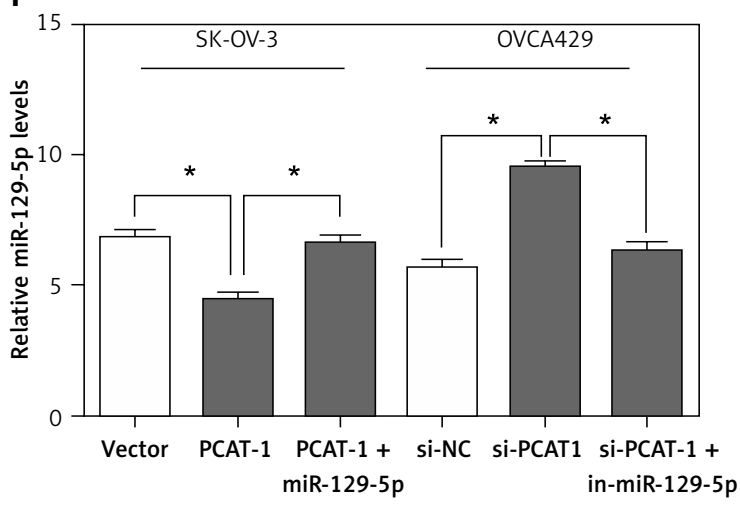

Figure 4. PACT-1 exerts its functions by directly targeting miR-129-5p in OC. A - Binding sites between PCAT-1 and miR-129-5p predicted by bioinformatics analysis. B - 293T cells were transfected with wild-type (WT) or mutated (mut) PCAT-1 reporter plasmids and miR-129-5p mimics (miR-129-5p) or miR-NC. The luciferase activities were measured by luciferase reporter assay. C - Expression of miR-129-5p in SK-OV-3 cells following PCAT-1 overexpression and OVCA429 cells following PCAT-1 knockdown. D - miR-129-5p restoration attenuated the effects of PCAT-1 overexpression on SK-OV-3 cell proliferation. E - miR-129-5p suppression abolished the effects of PCAT-1 knockdown on OVCA429 cell proliferation. F - miR-129-5p restoration or suppression attenuated the effects of PCAT-1 overexpression or knockdown on OC cell apoptosis

Data are mean $\pm S D .{ }^{*} P<0.05$ 
effects. These data reveal that PCAT-1 is an oncogene while miR-129-5p is a tumor suppressor in OC development.

Accumulating evidence indicates that IncRNAs always exert their functions by acting as ceRNAs which suppress the expression of target mRNAs via sequestering miRNAs. miR-129-5p was demonstrated as a tumor suppressor in various human cancers. In ovarian cancer, Tan et al. showed that miR-129-5p can repress the expression of YAP and TAZ expression so that the proliferation and tumorigenicity of OC cells can be inhibited [16]. Moreover, miR-129-5p was also regulated by many IncRNAs such as IncRNA MALAT1 in breast cancer and osteosarcoma $[17,18]$, and NEAT1 in hepatocellular carcinoma [19]. In our study, the bioinformatics prediction analysis indicated that PCAT-1 might interact with miR-129-5p. Data from the luciferase reporter assay and qRT-PCR demonstrated PCAT-1 can interact with and repress the expression of miR-129-5p in OC. miR-129-5p overexpression reversed the effects of PCAT- 1 on OC cell proliferation and apoptosis. These observations indicated that IncRNA PCAT-1 plays its roles by directly targeting and suppressing miR-129-5p in OC.

In conclusion, this study reported a novel InCRNA PCAT-1 involved in the development of ovarian cancer, which indicated that PCAT-1 may serve as a diagnostic marker in the detection of early OC patients and a therapeutic target in the treatment of OC. However, as the sample size was limited, we did not analyze the effect of PCAT-1 on OC patients' survival time, which is essential for PCAT-1 to act as a prognostic marker. In addition, it is well known that microRNAs always play their roles by binding to the 3'-untranslated regions (UTR) and repressing the expression of target mRNAs including miR-129-5p [20, 21]. We need further research to investigate the mechanism by which miR-129 exert its roles in ovarian cancer, so as to fully understand the biological functions of IncRNA PCAT-1 and miR-129-5 $p$ in the development and progression of OC.

Taken together, our results demonstrated that long non-coding RNA PCAT-1 is significantly upregulated in OC tissues and cell lines. PCAT-1 both promotes proliferation and inhibits apoptosis by directly targeting and suppressing miR-129-5p in OC cells. Silencing PCAT- 1 may be a novel therapeutic strategy in the treatment of OC.

\section{Acknowledgments}

Li-Ping Gu, Shuo Jin and Rong-Chun Xu contributed equally to this work.

\section{Conflict of interest}

The authors declare no conflict of interest.

\section{References}

1. Siegel R, Ma J, Zou Z, Jemal A. Cancer statistics, 2014. CA Cancer J Clin 2014; 64: 9-29.

2. Pongsavee M. Effects of 744ins 20 - ter240 BRCA1 mutation on breast/ovarian carcinogenesis and the role of curcumin in telomerase inhibition. Arch Med Sci Civil Dis 2017; 2: e125-9.

3. Jemal A, Bray F, Center MM, Ferlay J, Ward E, Forman D. Global cancer statistics. CA Cancer J Clin 2011; 61: 6990.

4. Edwards $H M$, Noer MC, Sperling CD, et al. Survival of ovarian cancer patients in Denmark: results from the Danish gynaecological cancer group (DGCG) database, 1995-2012. Acta Oncol 2016; 55 Suppl 2: 36-43.

5. Siegel R, Naishadham D, Jemal A. Cancer statistics, 2013. CA Cancer J Clin 2013; 63: 11-30.

6. Kung JT, Colognori D, Lee JT. Long noncoding RNAs: past, present, and future. Genetics 2013; 193: 651-69.

7. Feng $W$, Li L, Xu X, Jiao Y, Du W. Up-regulation of the long non-coding RNA RMRP contributes to glioma progression and promotes glioma cell proliferation and invasion. Arch Med Sci 2017; 13: 1315-21.

8. Prensner JR, Iyer MK, Balbin OA, et al. Transcriptome sequencing across a prostate cancer cohort identifies PCAT-1, an unannotated lincRNA implicated in disease progression. Nat Biotechnol 2011; 29: 742-9.

9. Bi M, Yu H, Huang B, Tang C. Long non-coding RNA PCAT-1 over-expression promotes proliferation and metastasis in gastric cancer cells through regulating CDKN1A. Gene 2017; 626: 337-43.

10. Wen J, Xu J, Sun Q, Xing C, Yin W. Upregulation of long non coding RNA PCAT-1 contributes to cell proliferation, migration and apoptosis in hepatocellular carcinoma. Mol Med Rep 2016; 13: 4481-6.

11. Qiao L, Liu X, Tang Y, Zhao Z, Zhang J, Feng Y. Down regulation of the long non-coding RNA PCAT-1 induced growth arrest and apoptosis of colorectal cancer cells. Life Sci 2017; 188: 37-44.

12. Fu LL, Li CJ, Xu Y, et al. Role of IncRNAs as novel biomarkers and therapeutic targets in ovarian cancer. Crit Rev Eukaryot Gene Expr 2017; 27: 183-95.

13. Li J, Huang H, Li Y, Li L, Hou W, You Z. Decreased expression of long non-coding RNA GAS5 promotes cell proliferation, migration and invasion, and indicates a poor prognosis in ovarian cancer. Oncol Rep 2016; 36: 3241-50.

14. Li H, LiU C, Lu Z, et al. Upregulation of the long non-coding RNA SPRY4-IT1 indicates a poor prognosis and promotes tumorigenesis in ovarian cancer. Biomed Pharmacother 2017; 88: 529-34

15. Wu L, Wang X, Guo Y. Long non-coding RNA MALAT1 is upregulated and involved in cell proliferation, migration and apoptosis in ovarian cancer. Exp Therap Med 2017; 13: 3055-60.

16. Tan G, Cao X, Dai Q, et al. A novel role for microRNA129-5p in inhibiting ovarian cancer cell proliferation and survival via direct suppression of transcriptional co-activators YAP and TAZ. Oncotarget 2015; 6: 8676-86.

17. Zuo Y, Li Y, Zhou Z, Ma M, Fu K. Long non-coding RNA MALAT1 promotes proliferation and invasion via targeting miR-129-5 $p$ in triple-negative breast cancer. Biomed Pharmacother 2017; 95: 922-8.

18. Liu K, Huang J, Ni J, et al. MALAT1 promotes osteosarcoma development by regulation of HMGB1 via miR-1423p and miR-129-5p. Cell Cycle 2017; 16: 578-87.

19. Fang L, Sun J, Pan Z, et al. Long non-coding RNA NEAT1 promotes hepatocellular carcinoma cell proliferation 
through the regulation of miR-129-5p-VCP-IkappaB. Am J Physiol Gastrointest Liver Physiol 2017; 313: G150-6.

20. Lan G, Yang L, Xie X, Peng L, Wang Y. MicroRNA-490-5p is a novel tumor suppressor targeting c-FOS in human bladder cancer. Arch Med Sci 2015; 11: 561-9.

21. Ma Z, Cai H, Zhang Y, Chang L, Cui Y. MiR-129-5p inhibits non-small cell lung cancer cell stemness and chemoresistance through targeting DLK1. Biochem Biophys Res Commun 2017; 490: 309-16. 